\title{
Risk Factors for Perinatal Asphyxia in Newborns Delivered at Term
}

\author{
Anália Luana Sena de Souza', Nilba Lima de Souza², Débora Feitosa de França ${ }^{3}$, \\ Samara Isabela Maia de Oliveira3 ${ }^{3}$ Anne Karoline Candido Araújo ${ }^{3}$, \\ Dândara Nayara Azevedo Dantas ${ }^{3}$

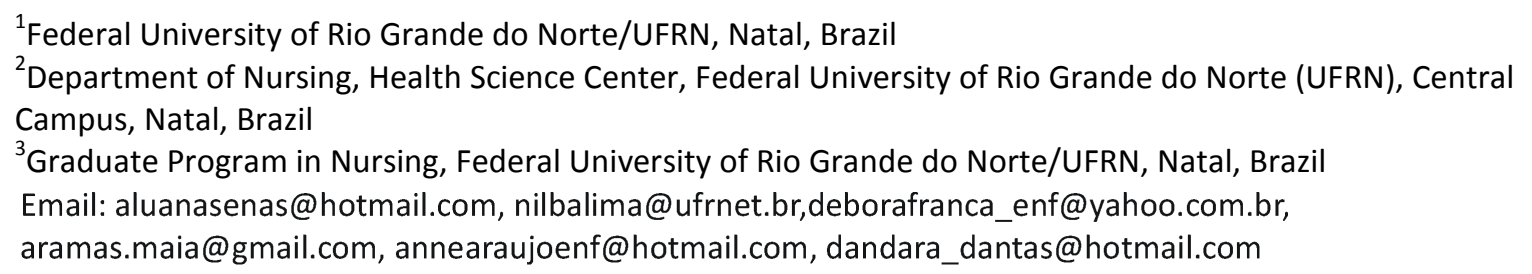

Received 28 May 2016; accepted 26 July 2016; published 29 July 2016

Copyright (C) 2016 by authors and Scientific Research Publishing Inc.

This work is licensed under the Creative Commons Attribution International License (CC BY). http://creativecommons.org/licenses/by/4.0/

(c) (i) Open Access

\begin{abstract}
Perinatal asphyxia is defined as harm to the fetus or the newborn caused by hypoxia and/or ischemia of various organs with intensity to produce biochemical and/or functional changes. Understanding the risk factors for this clinical condition allows the identification of vulnerable groups, enabling an improvement in care planning in the perinatal period in neonatal intensive care units. In this sense, this research aimed to identify risk factors for perinatal asphyxia present in newborns term that showed record for this clinical condition. This was a cross-sectional, retrospective documentary, quantitative and descriptive, conducted from data from medical records of 55 infants admitted to a neonatal intensive care unit. As for maternal characteristics (78.0\%) had between 16 and 35 years, only one child (53.0\%) and (76.0\%) had no prior history of miscarriage. As for pre-existing diseases or pregnancy (38.0\%) developed by Hypertensive Pregnancy Specific disease $(02.0 \%)$ were suffering from Hypertension and (02.0\%) of Diabetes Mellitus. As for newborns, most infants had birth weight (43.6\%) and correlation with gestational age (78.2\%) compatible for good conditions of birth. Only (20.0\%) of the infants had a difficult labor. It stood out although there was a slight predominance of severe asphyxia (50.9\%) in the first minute and (45.5\%) of the infants had record release intrauterine meconium. It was concluded that most mothers and newborns did not have risk factors for perinatal asphyxia, thus, this fact could be attributed to the structural conditions of service, especially in the care during labor, delivery and immediate assistance newborn.
\end{abstract}

\section{Keywords}

Birth at Term, Neonatal Asphyxia, Neonatal Intensive Care Units, Neonatal Nursing

How to cite this paper: de Souza, A.L.S., de Souza, N.L., de França, D.F., de Oliveira, S.I.M., Araújo, A.K.C. and Dantas, D.N.A. (2016) Risk Factors for Perinatal Asphyxia in Newborns Delivered at Term. Open Journal of Nursing, 6, 558-564. 


\section{Introduction}

Perinatal asphyxia is defined as a harm to the fetus or newborn (NB), caused by a lack of oxygen (hypoxia) and/or a lack of perfusion (ischemia) of various organs with sufficient intensity to promote changes in aerobic metabolism to anaerobic metabolism, triggering metabolic acidosis and cardiovascular decompensation, such as peripheral vasodilation and decreased cardiac output, resulting in severe fetal hypotension and reduced cerebral blood flow and consequently brain damage and organ dysfunction or fetal/neonatal death [1].

The parameter of the Apgar score is used to determine the level of birth asphyxia, evaluated in the first and fifth minutes of life, with scores ranging from zero to ten. Values obtained from four to seven in the first minute of life indicate birth asphyxia and the greater severity is between zero and three, suggestive of severe asphyxia [2].

Risk factors related to perinatal asphyxia are maternal age under 16 or over 35 years old; gestational age $<39$ or >41 weeks; arterial hypertension; diabetes; use of illicit drugs and alcohol; hypertensive disorders; premature rupture of the membranes; maternal infection; decreased fetal activity; bleeding in the second or third trimester; gestational age and weight discrepancy; stillbirth or earlier neonatal; fetal malformation; lack of prenatal care; abnormal pattern of fetal heart rate; cesarean section; general anesthesia; use of forceps or vacuum extractor; hypertonic uterine; non-cephalic presentation; meconium amniotic fluid; prolapse or cord rupture; cord knot; chorioamnionitis; use of opioids 4 hours before delivery; membranes rupture $>18$ hours; placental abruption; labor $>24$ hours; placenta previa and second stage of labor $>2$ hours [3].

Early neonatal mortality associated with perinatal asphyxia in newborns of low risk, that is, birth weight of $\geq 2500$ g and without congenital malformations, is high in our country. Between 2005 and 2010 in Brazil, there were 5 - 6 early deaths per day of low-risk newborns from causes associated with perinatal asphyxia. Brazil has achieved the Millennium Development Goal number 4, reducing child mortality. However, neonatal mortality is still $69 \%$ of infant deaths and $76 \%$ of deaths occurred 0 - 6 days after birth. Most of these deaths occurred on the first day of life-related to conditions of birth [3].

The evaluation of risk factors for asphyxia can help identify fetuses at risk, allowing proper reference within the health system, as well as the planning of assistance in the perinatal period in neonatal intensive care units, and the team of providence specializing in neonatal resuscitation. The care given to this newborn soon after birth will have a strong influence in reducing the serious consequences resulting from asphyxiation or even full recovery of the neonate.

Given the above and based on the experience of the daily life of the neonatal intensive care unit (NICU) of a maternity hospital, it was observed that perinatal asphyxia in its different degrees contributes as an important cause of hospitalization of infants, including term and post-term newborns.

It should be noted that when the NB is coming from a pregnancy considered the low risk with adequate birth weight and birth at term; it reduces the likelihood of suffering asphyxia. Thus, the question is: What are the risk factors for perinatal asphyxia present in term newborn infants with asphyxia record in a neonatal unit?

This study aims to identify risk factors for perinatal asphyxia present in term newborn infants with asphyxia record.

This study is justified by the interest of contributing to the health professionals and program substantiating their care interventions aimed at improving the quality of perinatal care and reduced neonatal mortality by asphyxia.

\section{Method}

This is a cross-sectional, retrospective documentary, quantitative and descriptive, carried out in a neonatal intensive care unit (NICU) of a maternity school, a reference to pregnancy and birth high risk, in the state of Rio Grande do Norte. The study population consisted of all infants born in that maternity, admitted to the NICU with registration for perinatal asphyxia.

The data collection period was between 2011-2013, for infants who had lower Apgar scores or equal to 7 at the first and fifth minutes of life, and gestational age at 37 weeks and 41 weeks and six days. These are the criteria for inclusion in the survey. The study excluded newborns with congenital malformations and syndromes, for being configured as confounding factors for the degree of perinatal asphyxia, by conditions related to pregnancy, labor, and birth.

The sample corresponded to the medical records of newborns included in the eligibility criteria. For the sample representativeness of the analysis, sample calculation was performed with the prevalence of perinatal anoxia 
of 3.67 [4] in newborns at term, with a confidence interval of $95 \%$ and sample loss of $10 \%$ needing to use a sample of 38 records. The initial quantitative sample regarding the medical records of newborns were 70 records. Of them, 15 were lost in the medical file and sector statistics (SAME). Thus, medical records of 55 infants were analyzed who were included in the final sample.

Data collection was in June and July 2015. The instrument used for data collection was built on the Neonatal Resuscitation Manual of the Brazilian Society of Pediatrics [5], which brings the risk factors for choking and composed four pieces that bring the variables to be studied: 1) maternal sociodemographic data; 2) data related to obstetric history, and pregnancy; 3) data related to labor and delivery; 4) data related to the conditions of birth and characterization of the NB.

The information collected in the study were entered into a specific database with the use of Microsoft Excel spreadsheets 2010 software, and further processed by the statistical program of free access, Statistical Package For Social Sciences (SPSS), version 20.0, and the data were analyzed using descriptive statistics using absolute and percentage frequencies.

The study was approved by the Ethics and Research Committee of the Federal University of Rio Grande do Norte, with CAAE 42792915.2.0000.5292 and obtaining the consent form of the institution.

\section{Results}

\subsection{Maternal Risk Factors, Obstetric History and Gestational Period}

Maternal characterization has a predominance of age between 16 and 35 years old (78.0\%). As for pre-existing diseases or pregnancy (38.0\%) developed Specific Hypertensive Pregnancy Disease (SHPD), (02.0\%) were suffering from Hypertension and (02.0\%) of Diabetes Mellitus. Of them, (53.0\%) were mothers for the first time and (76.0\%) had no prior history of miscarriage. All this is shown in Table 1.

In the previous delivery care (93.0\%) women held prenatal care. However, $(67.0 \%)$ had six or more visits. About the serology request for pregnancy, none of the women had antibodies to the human immunodeficiency virus (HIV) and only (02.2\%) presented reagent test for syphilis.

It is noteworthy that none of the progenitors had stillbirth history and (89.1\%) had positive Rh factor. The registration of complications during the pregnancy and puerperal period indicates that (3.6\%) had placental ab-

Table 1. Maternal characterization as the obstetrical and pregnancy risk factores. Natal, RN, Brazil, $2015(\mathrm{~N}=55)$.

\begin{tabular}{|c|c|c|c|}
\hline \multicolumn{2}{|c|}{ VARIABLES } & \multirow{2}{*}{$\begin{array}{c}\mathbf{N} \\
4\end{array}$} & \multirow{2}{*}{\begin{tabular}{|c|}
$\%$ \\
0.70
\end{tabular}} \\
\hline & Less than 15 years old & & \\
\hline Mothers'Age & Between 16 and 35 years old & 43 & 78.0 \\
\hline \multirow{3}{*}{ Pregnancy quantity } & Older than 35 years old & 8 & 15.0 \\
\hline & Primiparous & 29 & 53.0 \\
\hline & More than one pregnancy & 26 & 47.0 \\
\hline \multirow{2}{*}{ Prior miscariages } & No & 42 & 76.0 \\
\hline & Yes & 13 & 24.0 \\
\hline \multirow{2}{*}{ Hypertension } & No & 54 & 98.0 \\
\hline & Yes & 1 & 2.0 \\
\hline \multirow{2}{*}{ SHPD } & No & 34 & 62.0 \\
\hline & Yes & 21 & 38.0 \\
\hline \multirow{3}{*}{ Diabetes } & No & 53 & 96.0 \\
\hline & Yes & 1 & 02.0 \\
\hline & Ignored & 1 & 02.0 \\
\hline \multirow{2}{*}{ Oligoamnios } & No & 54 & 98.0 \\
\hline & Yes & 1 & 02.0 \\
\hline \multirow{2}{*}{ DPP } & No & 53 & 96.0 \\
\hline & Yes & 2 & 04.0 \\
\hline
\end{tabular}


ruption (DPP) and (1.8\%) oligoamnios. The obstetric data point to the mean gestational age at birth of 39 weeks and five days (SD \pm one week and three days).

\subsection{Risk Factors Related to Labor and Delivery}

It was found that the predominant type of Cesarean delivery was (61.8\%), the use of forceps was vaginally $(07.3 \%)$, and the use of any depressant drug of the central nervous system was $(14.5 \%)$, such as magnesium sulfate, opioids or benzodiazepines. The cephalic presentation was predominant (92.7\%) as well as ruptured membranes for less than six hours or broken in the labor act (80.0\%). As complications during labor and delivery, only (20.0\%) of the infants had a difficult labor, were no reports of difficulty related to the service (03.6\%) and (05.5\%) had other events. Of the total RN, (70.9\%) did not present any problem.

It is noteworthy that among caesarean deliveries $(n=34),(62.0 \%)$ were considered emergency $(94.0 \%)$ and were carried out by use of spinal anesthesia.

\subsection{Risk Factors Related to the Conditions of Birth and Characterization of the Newborn}

The variables of interest for the study of the conditions of birth analyzing the favorable factors for perinatal asphyxia are presented in Table 1 and Table 2.

As shown in Table 1, it is emphasized that most newborns had birth weight and correlation with gestational age matching good condition of birth and only (12.7\%) were classified as macrosomia and (16.4\%) as low birth weight, factors that favor the perinatal anoxia. For the presence of fetal suffering, only (09.1\%) of the infants had and (45.5\%) had record intrauterine meconium release.

As the level of asphyxia measured by Apgar, neonates born with Apgar 4 to 7 were considered mild to moderate asphyxia; and those born with Apgar scores of 0 to 3 as severe asphyxia. As noted in Table 1, it was detected that there was a slight predominance of severe asphyxia (50.9\%) in the first minute, but an evolution from mild to moderate in the fifth minute (89.1\%).

In the context of neonatal resuscitation, it is observed in Table 3, (94.5\%) of the infants required resuscitation,

Table 2. Characterization of term newborn infants who suffered perinatal asphyxia, as the conditions of birth. Natal, RN, Brazil, $2015(\mathrm{~N}=55)$.

\begin{tabular}{|c|c|c|c|}
\hline & VARIABLES & $\mathrm{N}$ & $\%$ \\
\hline \multirow{4}{*}{ Wight of the NB RN at birth } & Low weight at birth (<2.500 g) & 9 & 16.4 \\
\hline & Insufficient weight at birth (2.500 - 2.999 g) & 15 & 27.3 \\
\hline & Normal weight at birth (3 - 3.999 g) & 24 & 43.6 \\
\hline & Macrosomic (>4 g) & 7 & 12.7 \\
\hline \multirow{3}{*}{ Gender } & Male & 37 & 63.3 \\
\hline & Female & 18 & 32.7 \\
\hline & Suitable for Gestational Age & 43 & 78.2 \\
\hline \multirow[t]{2}{*}{ Size for gestational age } & Big for Gestational Age & 7 & 12.7 \\
\hline & Small for Gestational Age & 5 & 09.1 \\
\hline \multirow{2}{*}{ Suffering fetal intrauterine } & No & 50 & 90.9 \\
\hline & Yes & 5 & 09.1 \\
\hline \multirow{2}{*}{ Restriction of intrauterine growth } & No & 48 & 87.3 \\
\hline & Yes & 7 & 12.7 \\
\hline \multirow{2}{*}{ Intrauterine meconium release } & No & 30 & 54.5 \\
\hline & Yes & 25 & 45.5 \\
\hline \multirow{2}{*}{ Apgar $1^{\text {st }}$ minute } & Severe asphyxia & 28 & 50.9 \\
\hline & Mild to moderate asphyxia & 27 & 49.1 \\
\hline \multirow{2}{*}{ Apgar $5^{\text {th }}$ minute } & Mild to moderate asphyxia & 49 & 89.1 \\
\hline & Severe asphyxia & 5 & 10.9 \\
\hline
\end{tabular}


Table 3. Characterization of term newborn infants who suffered perinatal asphyxia, as the behaviors in the delivery room. Natal, RN, Brazil, $2015(\mathrm{~N}=55)$.

\begin{tabular}{cccc} 
VARIABLES & & $\mathrm{N}$ & $\%$ \\
\hline Resuscitation & Yes & 52 & 94.5 \\
Ventilation use with positive pressure (PPV) & No & 3 & 05.5 \\
and Oxygen & Yes & 47 & 85.5 \\
Orotracheal intubation (EOT) & No & 8 & 14.5 \\
Cardiac massage & Yes & 24 & 56.4 \\
Use of resuscitation drugs & No & 51 & 93.6 \\
& Yes & 4 & 07.3 \\
Meconium aspiration airway & No & 53 & 98.2 \\
& Yes & 1 & 01.8 \\
& No & 30 & 65.5 \\
\hline
\end{tabular}

most with the use of PPV with oxygen and (43.6\%) required EOT. They $(n=24)(67.0 \%)$ had severe asphyxia in the $1^{\text {st }}$ minute of life. Most cases did not require cardiac massage and the use of resuscitation drugs, but there was a record of meconium aspiration need for airway in (34.5\%) of NB, despite having been releasing intrauterine meconium in $(45.5 \%)$ cases.

\section{Discussion}

Maternal conditions and the period of pregnancy mainly affect neonatal mortality rates related to perinatal anoxia. Studies in Scotland and Brazil corroborate the findings in maternal age because the age group is between 16 - 34 years old and are primiparous. In this sense, although this range is considered low risk for complications during pregnancy and childbirth, it is believed that perinatal asphyxia in this segment of the population is a result of weakened actions related to monitoring of the pregnant woman hindering to detect the factors risk resulting in perinatal asphyxia [6] [7].

On this subject, another important factor is the prenatal care in the contribution of favorable perinatal outcomes. In this study, most women held prenatal with six consultations or more. Therefore, according to the health ministry, the number of consultations was considered appropriate. The presence of perinatal asphyxia, in this context, may indicate that complications during delivery would not be attributed only to prenatal care, but the care held by the hospital [8].

Regarding the type of delivery, most births occurred by cesarean delivery under spinal anesthesia, and this result is similar to a study that examined the prevalence of perinatal asphyxia in a reference maternity [4]. On the other hand, this information confronted with the findings of another study in which the perinatal asphyxia had more frequently in vaginal deliveries [9]. It is worth noting that the cesarean delivery between 37 and 39 weeks of gestation without antenatal risk factors for asphyxia, raises the chance of the need to perform resuscitation in newborns [3].

Regarding the clinical and/or obstetric complications in this study, only part of newborns released intrauterine meconium were aspirated. This situation was justified because there is meconium aspiration connector for the NB after birth and the vacuum being without working. This points the occurrence of failures in health care that provide a deficit in the quality of care in neonatal resuscitation caused by the lack of material available in the institution. Recently, the World Health Organization [10] launched a strategy for patient safety called the checklist of Safe Childbirth to reduce failures as those related to the lack of supplies available for care.

This problem is emphasized by authors attaching the lack of material and human resources as an obstacle to the proper neonatal care in the delivery room, especially in aspiration and resuscitation procedures which would be essential to minimize the consequences of perinatal asphyxia [11]. Thus, it is evident the importance of action planning, materials and care to lessen the level of perinatal asphyxia and promote the recovery of the neonate birth condition, improving the prognosis of this NB. 
In this context, it is noteworthy that the occurrence of asphyxia cases in NB with normal weight and gestational age at term may also be attributed to childbirth care. This care must be based on proper care at birth, use of strategy, as the partograph to monitor the labor and adequate resuscitation for newborns asphyxiated, as identified in this study the amount of $94.5 \%$ of the NB sample required resuscitation [12].

The need for positive pressure ventilation shortly after birth and the progressive improvement without resuscitation or endotracheal intubation in this study in some newborns points to the effectiveness of a good neonatal care in the delivery room. Other studies also emphasize that a good PPV, with correct positioning of the neck, is enough to solve asphyxia [3].

Thus, Brazil has sought to qualify care to the newborn including all professionals, forming a multidisciplinary health team to participate in a co-responsible way, since the hospitalization of pregnant women, ensuring adoption of appropriate technologies, the immediate host, companion of choice, midwife, free positioning, and among others, ensuring comfort, pain relief and safety, and avoiding situations such as perinatal asphyxia [13].

Regarding gender, in this study, there was a higher prevalence of males in the occurrence of perinatal asphyxia (63.3\%), a result similar to that found in a study in a NICU by searching for records [4]. However, another study showed a higher percentage to perinatal asphyxia in females [14].

Regarding the identification of asphyxia and its evolution, it was addressed in a study that the Apgar appears as an important indicator of this phenomenon. Thus, when the Apgar results in low scores, it can translate a fetal suffering and indicates that the baby suffered asphyxia, as shown in the findings of this study [15]. Moreover, the same study relates the fact that the low birth weight interferes directly in the NB compensation mechanisms, which are compromised, unlike the NB with a higher weight that can give better recovery mechanisms, despite the brain and heart diseases caused by perinatal asphyxia.

Other factors identified and related to asphyxia found in another study is the obstetric of pregnant history, as the performance of prenatal care (especially the number of consultations), the occurrence of clinical and/or obstetric complications, such as the most relevant: maternal age, premature birth, lower birth weight ( $<2500 \mathrm{~g})$, stillbirth history, primiparity, threat of premature birth and clinical complications [1].

In this perspective, a study conducted in Sweden points to the importance of health staff have knowledge about the risk factors related to perinatal asphyxia. In the survey, it was identified relevant factors for delivery care to minimize the consequences to the newborn and the mother, standing out: maternal age, women with infertility or sterility history, diabetes, twin pregnancies, pre- and post-term pregnancy and the estimated size of the fetus. Another issue is that in cases seen all asphyxia case should be worked as a source of learning for the team, enabling a targeted assistance needs of the NB [16].

Thus, it is essential that the risk factors identified and worked can be used as elements of planning, analysis and organization for care implemented to the newborn in cases of asphyxia. It was noted cases of asphyxia related to mortality rates in different realities, which directly reflects the type of assistance to this population. Therefore, it was perceived the relevance of the study on the issue proposed as interventions to that patient, who requires specific care.

\section{Conclusions}

This study concludes that, about the pregnancy risk factors, mothers of term newborn infants who suffer perinatal asphyxia do not have predominantly risk factors for this condition of birth related to maternal and gestational age, the number of prenatal visits and complications during pregnancy.

With regard to labor and childbirth, it is found that most are caesarean, considered urgent, which is a risk factor for neonatal asphyxia and it is found that among the complications of this period, some are related to the service, pointing to failure in the planning of actions and materials in the care of newborns in the delivery room.

Regarding the conditions of birth of the newborn, it was found that most needed resuscitation, using PPV with $\mathrm{O}_{2}$, but in some of the infants who showed releasing intrauterine meconium, the meconium aspiration of airways was not carried out by the lack of specific material for performing this procedure.

From the preceding, it is seen the need for adequacy of risk factors related to the occurrence of perinatal asphyxia in situations that go from the prenatal care of pregnant women, the delivery and conditions offered in health services.

In this sense, investing in training the staff to better assist the newborn at risk for neonatal asphyxia, given that complications may occur in the process, with action planning, materials, and care to lessen the level of perinatal asphyxia and ensure the rapid recovery of birth condition, improving the prognosis of the newborns who 
suffer asphyxia and possible consequences arising.

It is also noted the importance of the records, as this fact corroborated to limitations found during the study, as regards the absence of some data. It is necessary to identify the main risk factors involved in the genesis of perinatal asphyxia to improve the care to be directed to newborns in their respective service units. As suggestions, it is necessary to incorporate the need for care protocols for resuscitation situations.

It is noteworthy that there is difficulty in obtaining data relating to items of education of pregnant women, HbsAg serology for cytomegalovirus, toxoplasmosis and HCV, due to the failure on medical records filling.

\section{References}

[1] Takazono, P.S. and Golin, M.O. (2013) Perinatal asphyxia: Repercussions Neurological Disorders and Early Detection. Magazine. Neuroscience, 21, 108-117.

[2] International Classification of Diseases-ICD 10 (1993) ICD-10. International Classification of Diseases and HealthRelated Problems SUS Department of Informatics.

[3] Almeida, M.F.B., Ginsburg, R. and Anchieta, L.M. (2016) Neonatal Resuscitation: Guidelines for Health Professionals. Brazilian Society of Pediatrics, Rio de Janeiro.

[4] Silva, D.A., Costa, R.C.C. and Gonzaga, C.I.A. (2014) Perinatal Asphyxia: Prevalence and Risk Factors in Newborns at Term. Interdisciplinary Journal, 7, 134-140.

[5] Brazilian Society of Pediatrics (2015) Neonatal Resuscitation: Guidelines for Health Professionals. 5th Edition, SBP, Rio de Janeiro.

[6] Daripa, M., Caldas, H.M., Flores, L.P.O., Waldvogel, B.C., Ginsburg, R. and Almeida, M.F.B. (2013) Perinatal Asphyxia Associated with Early Neonatal Mortality: A Population Study of Preventable Deaths. Paulista Journal of Pediatrics, 31, 37-45. http://dx.doi.org/10.1590/S0103-05822013000100007

[7] Sharma, V., Lakshminrusimha, S., Carrion, V. and Mathew, B. (2015) Resuscitator's Perceptions and Time for Corrective Steps Ventilation during Neonatal Resuscitation. Resuscitation, 91, 63-66. http://dx.doi.org/10.1016/j.resuscitation.2015.03.008

[8] Domingues, R.M.S.M., Hartz, Z.M.A., Days, M.A.B. and Leal, M.C. (2012) Evaluation of Adequacy of Prenatal Care in the SUS in the City of Rio de Janeiro, Brazil. Public Health Notebook, 28, 425-437.

[9] Cross, A.C.S. and Ceccon, M.E.J. (2010) Prevalence of Perinatal Asphyxia and Hypoxic-Ischemic Encephalopathy in Term Newborns Considering Two Diagnostic Criteria. International Journal Human Growth and Development, 20, 302-316.

[10] WHO (2015) Safe Childbirth Checklist Implementation Guide: Improving the Quality of Delivery-Based Facility for Mothers and Newborns. Boston, 300.

[11] Mendonça, S.D., Medeiros, V.G.O., Souza, N.L., Silva, R.K.C. and Oliveira, S.I.M. (2015) Meconium Aspiration Syndrome: Identifying Obstetric and Neonatal Risk Situations. Care Research Magazine Is Fundamental Online, 7, 2910-2918.

[12] Mmbaga, B.T., Lie, R.T., Olomi, R., Mahande, M.J., Olala, O. and Daltveit, A.J. (2012) Causes of Perinatal Death at a Tertiary Care Hospital in Northern Tanzania 2000-2010: A Study Based Registry. Pregnancy and Childbirth, 12, 2-9. http://dx.doi.org/10.1186/1471-2393-12-139

[13] Lansky, S., Friche, A.A.L., Silva, A.A.M., Campos, D., Bittencourt, S.D.A., Carvalho, M.L., Frias, P.G., Cavalcante, R.R. and Cunha, A.J.L.A. (2014) Search Sunrise in Brazil: Profile of Neonatal Mortality and Evaluation of the Assistance to the Mother and Newborn. Public Health Notebook, 30, 192-207.

[14] Kings, L.A., Santos, C.V., Britto, T.I., Botelho, S.M., Jesus, C.S. and Santos, W.S. (2009) Epidemiological Analysis of Perinatal Asphyxia in Newborns at the General Hospital Prado Valadadres (HGPV). Magazine Baiana of Public Health, 33, 311-322.

[15] Oliveira, T.G., Freire, P.V., Moreira, F.T., Moraes, J.S.B.F., Arrelaro, R.C., Rossi, S., Ricardi, V.A., Julian Y., New, N.F. and Bertagnon, J.R.D. (2012) Score of Apgar and Neonatal Mortality in a Hospital Located in the Southern Area of São Paulo. Einstein, 10, 22-28. http://dx.doi.org/10.1590/S1679-45082012000100006

[16] Berglund, S. (2011) Every Case of Asphyxia Can Be Used as a Learning Example. Journal Perinatal Medicine, 40, 918. 


\section{Submit or recommend next manuscript to SCIRP and we will provide best service for you:}

Accepting pre-submission inquiries through Email, Facebook, LinkedIn, Twitter, etc.

A wide selection of journals (inclusive of 9 subjects, more than 200 journals)

Providing 24-hour high-quality service

User-friendly online submission system

Fair and swift peer-review system

Efficient typesetting and proofreading procedure

Display of the result of downloads and visits, as well as the number of cited articles

Maximum dissemination of your research work

Submit your manuscript at: http://papersubmission.scirp.org/ 